\title{
A Hybrid Chebyshev-ICA Image Fusion Method Based on Regional Saliency
}

\author{
Zaid Omar*, Tania Stathaki, Musa M. Mokji, Lila Iznita Izhar \\ Faculty of Electrical Engineering, Universiti Teknologi Malaysia, 81310 Johor Bahru, Malaysia \\ Corresponding author, e-mail: zaid@fke.utm.my
}

\begin{abstract}
An image fusion method that performs robustly for image sets heavily corrupted by noise is presented in this paper. The approach combines the advantages of two state-of-the-art fusion techniques, namely Independent Component Analysis (ICA) and Chebyshev Poly-nomial Analysis (CPA) fusion. Fusion using ICA performs well in transferring the salient features of the input images into the composite output, but its performance deteriorates severely under mild to moderate noise conditions. CPA fusion is robust under severe noise conditions, but eliminates the high frequency information of the images involved. We pro-pose to use ICA fusion within high activity image areas, identified by edges and strong textured surfaces and CPA fusion in low activity areas identified by uniform background regions and weak texture. A binary image map is used for selecting the appropriate method, which is constructed by a standard edge detector followed by morphological operators. The results of the proposed approach are very encouraging as far as joint fusion and denoising is concerned. The works presented may prove beneficial for future image fusion tasks in real world applications such as surveillance, where noise is heavily present.
\end{abstract}

Keywords: Image and data fusion, Chebyshev polynomials, independent component analysis, regionbased fusion

Copyright $\odot 2017$ Universitas Ahmad Dahlan. All rights reserved.

\section{The Basics of Image Fusion}

In image processing and computer vision, fusion is the process of combining relevant information from two or more image inputs into a single image. The goal is that the resulting composite should be more informative and has a higher perceptual and objective quality than any of the input images. Recent advances in image fusion are mainly due to the increasing availability of imaging sensors, as for example space-borne sensors. Popular fusion methods today include Independent Component Analysis (ICA) [2], by which an image region consisting of several adjacent pixels usually located within a squared-shape neighbourhood, may be decomposed into a linear combination of patches-similar in theory to the Fourier series. The name derives from the assumption that the patches are independent and thereby treated as random variables that are as non-Gaussian as possible [2]. Fusion is performed in the ICA domain by a combination of the corresponding coefficients of the input image bases. ICA is generally an excellent fusion method in non-noisy conditions, particularly due to its ability to preserve edges and gradient saliency within images.

More recently, a fusion method employing Chebyshev Polynomial Analysis (CPA) was introduced in [1] which resembles a low-pass filter. The method assumes the orthogonal polynomials generated from the two-dimensional Chebyshev series expansion as a new set of basis functions where the coefficients are fused. This is especially useful for denoising by which CPA fusion impulsively purges high frequency noise components through a careful selection of the polynomial degree utilised.

\subsection{Fusion for Image Denoising}

One major concern of fusion is the fact that real-life image acquisition applications are often susceptible to noise, therefore prone to corruption and error. In order to remove the noisy components, a method is required to recognise and subsequently transfer only the salient features of the image inputs into a composite output image. This is performed by certain fusion 
algorithms, which are able to estimate a denoised image of acceptable quality, with the least amount of transferred noise possible.

A standard approach to achieve joint fusion and denoising is to use an energy compact model, such as those in the transfer domain, whereby only prominent basis components are retained. To date there exist few techniques to specifically address this. ICA, via Sparse Code Shrinkage (SCS), has been successfully tested for denoising on several occasions but suffers from the limitation of estimating the noise variance beforehand [10]. SCS can similarly be generalised to most other transform domain methods to handle noise. CPA on the other hand is a type of low-pass filter that removes noise in heavily corrupted images without estimating its variance, though at a cost of lower signal accuracy [1].

This gives rise to a new type of fusion technique, which can be viewed as an algorithm fusion process that manages to combine the best aspects of both ICA and CPA. In this paper an image fusion approach is proposed for the denoising of digital images corrupted with noise. We provide a new technique for image fusion that complements the best aspects of both ICA and CPA, which we call the Hybrid fusion method [11].

\subsection{A Proposed Region-Based Approach}

The main aspect of Hybrid fusion is detecting edges, boundaries and texture. This is achieved by dividing the pixels of a given image into active and inactive regions, in other words distinguishing edges and rich texture as opposed to uniform background or weak texture. For this task, we employ the Canny edge detection method. The primary purpose of classification is to create a boundary among regions which correspond to different aspects of an image. Fusion is then applied individually on each bounded region.

There are two main attractions of this technique: pixels can be processed more efficiently if they are treated as a collective group within a region, rather than separate entities. Region-based fusion may therefore help to overcome some drawbacks of pixel-based fusion, like blurring, susceptibility to noise and misregistration [12]. The second advantage is that this approach enables us to perform image fusion using a mixture of CPA and ICA, thus getting the best of both approaches.

\section{Algorithms Comprising Hybrid Fusion}

In this section we discuss the methods that make up our proposed technique, namely ICA and CPA. The basic properties of each method are presented, along with their advantages. A detailed comparison has also been made between ICA and CPA is relevant aspects.

\subsection{Introduction to Independent Component Analysis}

Independent Components Analysis (ICA) is a popular method for distinguishing underlying components in a random data set [13]. The rationale behind ICA may be explained by the central limit theorem, whereby the linear mixture of two non-Gaussian and independent source signals results in a signal that is more Gaussian-like than its sources. ICA searches through the given mixed signal to precisely identify the most independent components - which are defined to be the source signals. Given a number of observed mixed signals $x$, the ICA attempts to identify the source signals $\mathrm{s}$, by means of deriving a mixing matrix $A[10]$ through the Equation $x=A s$.

We solve for $A$ by optimising the objective function of the distribution pertaining $s$, either by maximising its non-Gaussianity or minimising the mutual information between the independent components. Further, a study by Hoyer and Hyvarinen [15] noted that performing ICA on a given set of image patches will yield a number of very localised, independent components that tend to resemble the human's visual cortex in analysing scenery and detecting edges. They are also very similar to wavelet bases, and are therefore suitable for image analysis applications [16]. The aim is thus to estimate a finite number of bases that are able to represent most of the image patch's structure.

The new domain is described by a set of $2 \mathrm{D}$ basis functions (basis images). The image of interest is then represented as a linear combination of these bases. Bases are trained from a selection of varying image patches, belonging to training images that are similar in content and type to the complex set of images of interest. Estimation and optimisation methods are then employed to solve the mixing matrix and subsequently generate the source signals via the 
equation $y=W x$ where $W$ is the inverse of $A$, and $y$ is the derived signal aimed to approximate the source $s$.

Using the kurtosis method in practice, we begin by defining an arbitrary value for $W$ and calculate the direction in which the kurtosis of $y=W x$ is increasing the most, based on the available samples of the observed vector $x$. A gradient method is then used to find a new vector $W$. The process is reiterated until a convergence criterion is satisfied and the source signal's kurtosis is found. Thus theoretically, kurtosis can be used as an optimisation tool to address the ICA problem [2]. For a more detailed description of ICA, $[10,2,17]$ are excellent references.

\subsection{Introduction to Chebyshev Polynomials Analysis}

One-dimensional Chebyshev Polynomials, written mathematically as $T_{n}(x)$ can be defined via the recursive equation

$$
T_{0}(x)=1, T_{1}(x)=x, T_{\mathrm{n}+1}(x)=2 x T_{\mathrm{n}}(x)-T_{\mathrm{n}-1}(x)
$$

whereby their properties have been explained in [19]. For one-dimensional signal approximation, the polynomials can be used to estimate a given signal $f(x)$ :

$$
\tilde{f}(x)=\sum_{n=0}^{N-1} a_{n} T_{n}(x)
$$

where $\tilde{f}(x)$ is the approximation, and an a coefficient on $\mathrm{n}$ which was proven to have the following form [1]:

$$
a_{n}=\frac{2}{\pi} \sum_{x=-1}^{1}(1-x)^{-\frac{1}{2}} f(x) T_{n}(x)
$$

The Chebyshev polynomials are sorted based on their order. Thus a finite order $n$ used in CPA expansion enables basic signal features to be retained while more complex polynomials can be omitted. In contrast, ICA decom-poses a signal into a set of equally independent basis components which are randomly arranged. The difference is evident in performance evaluation of non-noisy images whereby ICA outperforms CPA considerably due to its ability to detect and enhance strong, independent features within a given scene. However due to the same reason, CPA per-forms better than ICA when tested under noisy conditions [1]. The concept of CPA can be generalised to other signal decomposition approaches whereby a finite number of bases are acquired and used to adequately represent a signal.

A separable extension of 1D CPA, similar to the discrete cosine transform (DCT), was subsequently intro-duced for use on image signals, called two-dimensional separable Chebyshev Polynomials. Its definition and proper-ties are given below [1]:

$$
\tilde{f}(x, y)=\sum_{m=0}^{M-1} \sum_{n=0}^{N-1} a_{m, n} T_{m}(x) T_{n}(y)
$$

and the coefficient $a_{m, n}$ is given by

$$
a_{m, n}=\frac{4}{\pi^{2}} \sum_{x=-1}^{1} \sum_{y=-1}^{1}(1-x)^{-\frac{1}{2}}(1-y)^{-\frac{1}{2}} f(x, y) T_{m}(x) T_{n}(y)
$$

For corrupted images, noise components tend to mostly occupy the higher frequency spectrum. Incidentally, as higher order polynomials comprise of high frequency components, the idea therefore is to limit the CPA order so as to remove noise components at a cost of also removing high energy information, including edges and strong texture. Effectively, CPA approximation acts as a low-pass filter that eliminates unwanted noise at the expense of lower signal accuracy. To extend this useful feature to fusion applications, comparisons are made between image/data coefficients as done in $[1,20]$. 


\section{Hybrid Fusion Framework}

\subsection{Region-based fusion}

In region-based schemes, pixels are segmented into larger regions. Each region is formulated to be based on such criteria as 'objects of interest' or 'degree of activity'. We are then able to determine the actual contribution of regions from each input. There has been numerous researches involving region-based fusion as can be found in [3], [12], [21].

We define an image or scene as being composed of active and inactive regions. A region is considered to be active if and only if there happens to be sufficient amount of 'interesting' information within, such as edges or strong texture. On the other hand, inactive regions imply a lack of interesting information or a plain area with weak textural details.

In images corrupted with noise, the presence of noise is prone to spread through the entire image, triggering a notable increase in the overall pixel activity. Our premise here is that noise is more distinguishable, and therefore more easily suppressed, in inactive regions than active ones. Noise components near an edge region, for example, would be mixed with the textural details associated to that edge. It would therefore be difficult to differentiate and subsequently remove noise without affecting the regional texture. It is due to this that blurring tend to occur in some image denoising applications such as Chebyshev Polynomials.

Of the previous fusion methods, ICA is extremely adept at modelling edges and textured regions by virtue of its ability to capture essential image features comprehensively. However, despite its denoising capability by use of sparse-code features [14], the performance of ICA has been shown as being prone to decline especially in very noisy examples. This has been highlighted in [1] and also in [11]. In contrast, the smoothness property of CPA makes it better suited to filter noisy signals, and as such would be of convenient use on inactive regions mentioned above.

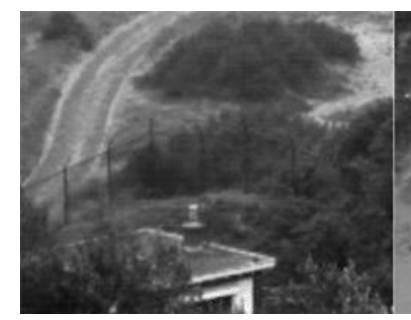

(a)

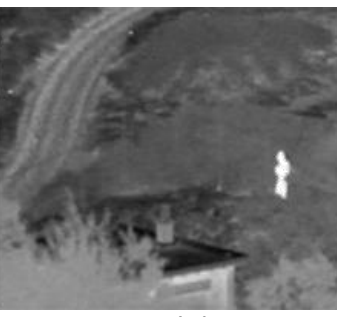

(b)

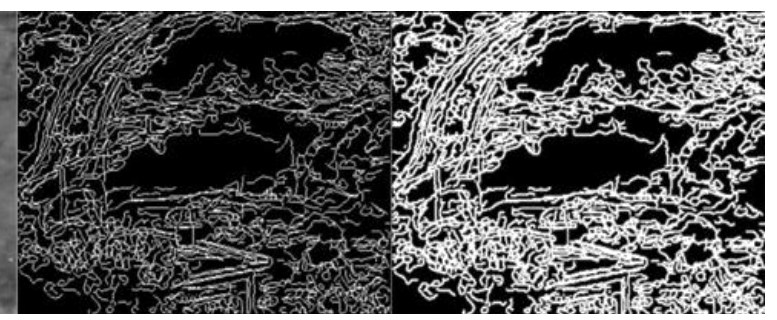

(c) (d)

Figure 1. Edge map derived from selecting all hard and soft edges from both input images (a) UN visual camera, (b) Infrared, (c) Edge map, (d) Enhanced edge map

The ideal solution in dealing with noisy scenarios would therefore be to use ICA on active regions which contain important structural image details, whilst inactive or background regions are processed by CPA. We will attempt to implement this idea in the paper. In a way, we are performing a second stage fusion on the results of the fusion methods that were initially obtained. The method is flexible enough for us to replace ICA and CPA with a variety of other methods; though for the purpose of denoising, the aforementioned methods are appropriate.

\subsection{Determining active and inactive regions}

Two input images from the UN Camp sequence database i.e. in Figure 1 are set as examples of the region defining process. Both images are processed by Canny edge detection, which is mostly robust to noise and is essentially a multistage edge detection algorithm that locates critical edge pixels at various scales. The filtered product, named the edge map, is meant to contain salient information regarding the image. The respective edge maps for the inputs are then combined via the logical $O R$ operator to form a single, global edge map. This step aims not to accurately determine correct edges or regions from the source images, but rather to ensure all possible edges and regions have been preserved for further processing [16]. This is similar to a scheme proposed by Drajic in [16] and [22], where active regions are defined 
by an activity indicator, $E(t)$, of an $N \times N$ image patch. A region is considered active if $E(t)>$ $\frac{2}{T} \sum_{k=1}^{T} E_{k}(t)$.

The resulting map is shown in Figure 1. The enhanced edge map provides a guide of the areas to be processed by either ICA or CPA. Active regions, denoting all hard edges and texture are represented by white pixels and will be fused by ICA whereas inactive regions i.e. those in black are fused by CPA.

However this is an overly simple model - blocking artefacts and pixel discontinuities tend to appear in the result which require refining. The enhanced process comprises a morphological opening step, as explained below.

\subsection{Mathematical Morphology for Edge Map Enhancement}

To preserve edge details, an intermediary step comprising an opening morphological operation is added to the edge map that will incorporate more pixels from ICA. Opening is a combination of morphological operations that consists of dilating an image after it has been eroded. The appeal of this step is that it discards small pixel artefacts and discontinuities, whilst smoothing larger pixel structures. In reference to the edge map, the opening step will suppress any single active pixel or small active region while at the same time increase the breadth of larger active regions. This is clearly evident in Figure 1d where the edges have been thickened, as compared to the original edge map in Figure 1c.

The map also illustrates inactive areas which form the majority of the image space. This maneuver allows us to maximise the use of CPA for autonomous noise suppression on the majority of the scenery, and at the same time enables ICA to process edge lines and hard textural regions which represent important features in an image. In general the additional step provides a clearer and more balanced usage ratio between pixels belonging to ICA and CPA

In practice, consider two output images of ICA and CPA, $F_{I C A}$ and $F_{C P A}$ respectively. The edge map is a binary map with binary values, $S \in\{0,1\}$ with white being 1 and black being 0 . The expression used to derive Hybrid fusion, $F_{\text {Hybrid }}$ can be written as a Hadamard product,

$$
F_{\text {Hybrid }}=\left(F_{I C A} \circ S\right)+\left(F_{C P A} \circ \bar{S}\right)
$$

Table 1. Average Petrovic score i.e. $E(Q)$ for fusion experiments at noise levels $16 \mathrm{~dB}$ to $10 \mathrm{~dB}$. Comparisons between ICA, CPA and Hybrid fusion.

\begin{tabular}{llrrrrl}
\hline Image set & \multicolumn{2}{l}{ Clock } & \multicolumn{3}{c}{$3 \mathrm{M}$} & \multicolumn{2}{c}{ Desk } & Weighted \\
Fusion method & Max-abs & Weighted & Max-abs & Weighted & Max-abs & Wein \\
\hline ICA & 0.2893 & 0.3293 & 0.4860 & 0.5048 & 0.4165 & 0.4189 \\
CPA & 0.3144 & 0.3342 & 0.4954 & 0.4875 & 0.3926 & 0.3937 \\
Hybrid fusion & 0.3174 & 0.3453 & 0.5109 & 0.5076 & 0.4269 & 0.4117 \\
\hline
\end{tabular}

$$
F_{\text {Hybrid }}=\sum_{x=1}^{X} \sum_{y=1}^{Y}\left[F_{I C A}(x, y) \cdot S(x, y)+F_{C P A}(x, y) \cdot \bar{S}(x, y)\right]
$$

where $x=1,2, \ldots, X$ and $y=1,2, \ldots, Y$ are the pixel coordinates and $(X \cdot Y)$ is the image size.

The concept brought forth in this paper is similar to the way humans generally perceive objects of interest within our line of vision. Uniform areas in the background tend to be plain and carry little or no information of high frequency. The general appeal of our method is it can enhance strong texture and edges, thus giving priority to meaningful regions whilst deprioritising the less important regions in the background.

\section{Performance Evaluation of Hybrid Fusion}

We tested our algorithm using corrupted images, to reflect real world conditions whereby the transmission of data may be prone to noise. Incremental Gaussian noise was added to multiple sets of input images, ranging from $16 \mathrm{~dB}$ to $10 \mathrm{~dB}$ to represent the various degrees of image corruption. Scenarios were used from the multifocal Clock, 3M and Desk: all of which are common image datasets for evaluation of fusion performances. For the experiment, 
we obtained two grayscale images as source signals, from which the fusion will generate a composite output image. For the sake of brevity only the Clock experiment is illustrated, as shown in Figure 2a.

For benchmarking purposes, we tested the performance of Hybrid fusion against its predecessor methods, namely ICA and CPA. ICA training was carried out on 1000 patches belonging to images of similar content, each patch measuring $7 \times 7$ pixels. A total of 48 independent bases were derived. After several configurations and experiment, we concluded that the size and number of patches are large enough without being overly complex, and presents a good balance between information content and computational complexity. Similarly for CPA, $m=11$ and $n=11$ degree of Chebyshev polynomials were chosen and $7 \times 7$ overlapping patches were used. Overlapping is performed by a shift of one pixel per iteration. All fusion methods utilise the max-abs and weighted average fusion rule [3].

In non-noisy environments, CPA's approximation results in a smooth fusion with less discernible edges. CPA filters out most high frequency components, creating a blurred artefact on the image. Yet through the incremental in-clusion of noise elements, the image quality only degrades slightly as has been shown in [1]. This may be explained by the universal property within CPA that automatically removes additive noise and other components of high frequency. The limitation of CPA however is that it does not yet solve the problem of blurred edge details.

Therefore a combined output containing the best aspects of the above two methods is desired. An output image should clearly detect and differentiate edges and important texture, whilst at the same time adequately suppress the noise. The Hybrid method is able to accomplish this by combining edge and textural regions from ICA and background regions from CPA [11]. The result is an enhanced output with distinctively better subjective visual quality and higher scores in objective evaluation.

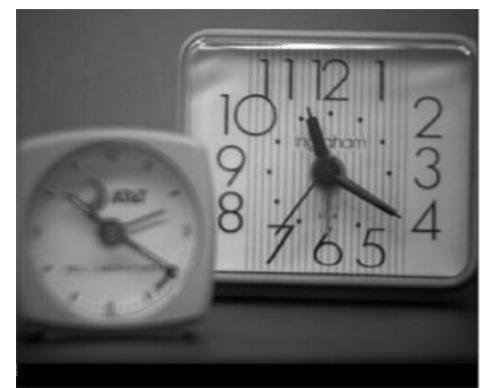

(a)

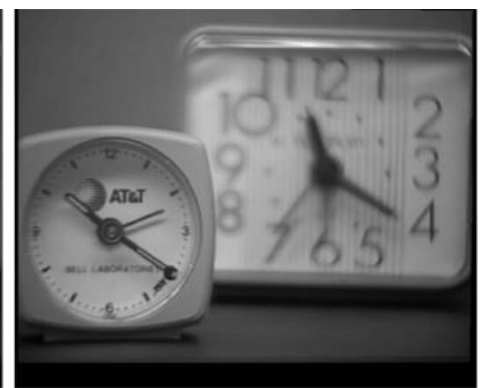

(b)

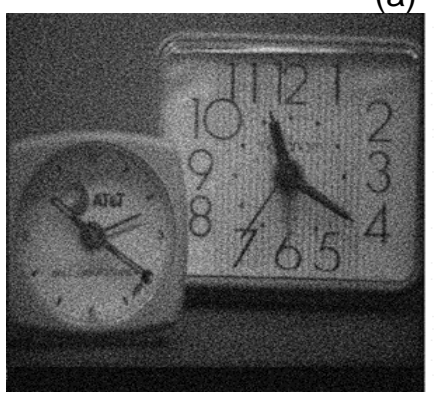

(c)

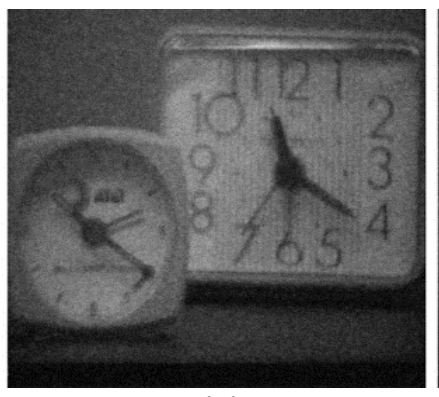

(d)

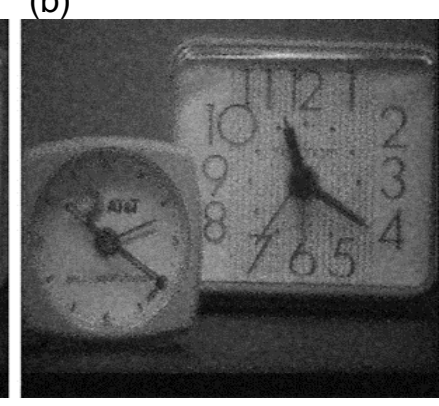

(e)

Figure 2. Multifocal Clock image fusion results for different methods at $\mathrm{SNR}=10 \mathrm{~dB}$ using Maxabs rule. a) Clock 1 (background focused), b) Clock 2 (foreground focused), c) ICA, d) CPA, e) Hybrid fusion

The objective image fusion performance measure [23], also called the Petrovic metric, assesses the quality of visual information obtained from the fusion of input images. The metric works by extracting all perceptually important information in the inputs, and subsequently measure quantitatively the ability of the fusion method to transfer this information to the output image. The metric attributes salient information with the edge strength and orientation of each 
pixel, which are derived by the Sobel operator. A normalised weighted performance metric $Q^{A B / F} \in[0,1]$ is used to measure fusion performance. In the experiment, two fusion rules were used - max-abs and weighted average. Employing the Petrovic metric [23] on our set of images produces the following results in Table 1. The table displays the average Petrovic score, or $E\left(Q^{A B / F}\right)$ over SNR levels $16 \mathrm{~dB}$ to $10 \mathrm{~dB}$ for each fusion method. An observation of the scores confirm that Hybrid fusion has the best performance of the three.
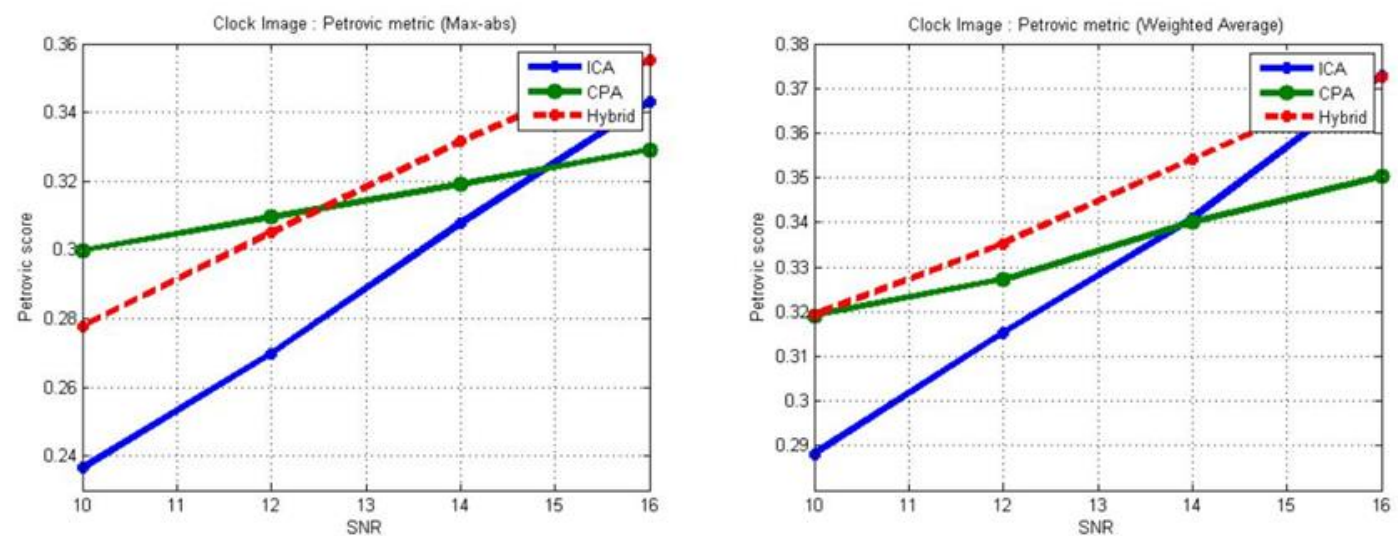

Figure 3. Multifocal Clock fusion performance for different fusion methods for $\mathrm{SNR}=16 \mathrm{~dB}$ to 10dB. a) Max-abs, b) Weighted average fusion rule. (ICA=blue, CPA=green, Hybrid=red)

The graphs displaying the performance of each method under the different noise levels is given in Figure 3. The graphs for CPA depict a very low gradient against the rising level of noise. The reason behind this has been discussed earlier in the section. In contrast, ICA tends to suffer drastically as the noise increases. In contrast, Hybrid fusion almost always retains the highest score. In the case of Clock and ' $3 \mathrm{M}$ ', Hybrid fusion scores have consistently been the highest throughout all SNR levels. The promising results of Hybrid fusion are likely due to the combinatory factor of the technique. Strong edge details belonging to ICA in active regions have been retained while inactive and plain regions have been fittingly smoothed.

\section{Conclusion}

We have described a novel combinatory method for fusing images and have successfully tested Hybrid fu-sion's ability alongside existing methods. In our tests we have looked into fusing two grayscale images. The positive results are proof that our approach is valid and meaningful. The final output was derived by a piece-wise composition of two prominent fusion techniques: ICA and CPA. It is therefore entirely possible to extend the scope of our applica-tion to three or more methods, and not necessarily limited to the aforementioned two. Our original aim was to generate the best possible image from a higher-level fusion step. This is a feasible direction in which to take our research.

Hybrid fusion can essentially be defined as a regional composite of the fusion results of ICA and CPA, whereby selected pixels of ICA and CPA are injected into an image framework based on the regions from the edge map. The criteria for selection is defined according to active regions, translated as areas with edge and textural details, and inactive regions. It is due to this therefore that the findings are positive; and Hybrid fusion is able to score higher than other methods when tested using the objective fusion measure. The work presented here introduces us to new possibilities for improvements especially in the sub-area of edge and saliency-based image fusion.

\section{References}

[1] Z Omar, N Mitianoudis, T Stathaki. Two-dimensional Chebyshev polynomials for image fusion, 28th Picture Coding Symposium, Nagoya. 2010.

TELKOMNIKA Vol. 15, No. 2, June 2017: $934-941$ 
[2] A Hyvarinen, E Oja, Independent component analysis: algorithms and applications. Neural Networks. $2000 ; 13(4-5): 411-430$.

[3] T Stathaki. Image Fusion: Algorithms and Applications, Edited Book, Academic Press, 500. 2008.

[4] P Hill, N Canagarajah, D Bull. Image Fusion using Complex Wavelets. Proceedings of the 13th British Machine Vision Conference, Cardiff, United Kingdom. 2002.

[5] www.metapix.de/methods.htm, accessed 3/7/2011.

[6] F Nencini, A Garzelli, S Baronti, L Alparone. Remote sensing image fusion using the curvelet transforms, Information Fusion. 2007; 8: 143-156.

[7] M Choi, RY Kim, MR Nam, HO Kim. Fusion of multispectral and panchromatic satellite images using the curvelet transform, IEEE Geoscience and Remote Sensing Letters. 2005; 2(2): 136-140.

[8] JL Starck, EJ Candes, DL Donoho. Gray and color image contrast enhancement by the curvelet transform. IEEE Transaction on Image Processing. 2003; 12(6): 706-717.

[9] H Hariharan, A Gribok, B Abidi, M Abidi. Multi-modal face image fusion using Empirical Mode Decomposition, Biometric Consortium Conference, Virginia, USA. 2005.

[10] DP Acharya, G Panda. A review of independent component analysis techniques and their applications. IETE Technical Review. 2008; 25(6).

[11]Z Omar, N Mitianoudis, T Stathaki. Region-based image fusion using a combinatory Chebyshev-ICA method. Proc. Intl. Conf. Acoustics, Speech and Signal Processing, Prague. 2011; 1213-1216.

[12] G Piella. A Region-based Multiresolution Image Fusion Algorithm. ISIF Fusion Conference 2002, Annapolis. 2002.

[13] JV Stone. Independent component analysis: an introduction, TRENDS in Cognitive Sciences. 2002; 6(2): 59-64.

[14]E Oja, A Hyvarinen, P Hoyer. Image Feature Extraction and Denoising by Sparse Coding, Pattern Analysis \& Applications. 1999; 2: 104-110.

[15] A Hyvarinen, PO Hoyer. Emergence of phase and shift invariant features by decomposition of natural images into independent feature subspaces, Neural Computation. 2000; 12(7): 1705-1720.

[16]N Mitianoudis, T Stathaki. Pixel-based and Region-based Image Fusion schemes using ICA bases. Information Fusion. 2007; 8(2): 131-142.

[17] A Hyvarinen. Fast and robust fixed-point algorithm for independent component analysis. IEEE Transactions on Neural Networks. 1999; 10(3): 626-634.

[18] A Hyvrinen, P Hoyer, E Oja. Image Denoising by Sparse Code Shrinkage. In S. Haykin and B. Kosko (eds), Intelligent Signal Processing, IEEE Press. 2001

[19] JC Mason, DC Handscomb. Chebyshev Polynomials, Chapman \& Hall/CRC, Florida. 2003; 105-141.

[20] N Amthul. Image fusion using two dimensional Chebyshev polynomials, MSc Dissertation. Imperial College. London. 2009.

[21] N Cvejic, J Lewis, D Bull, N Canagarajah. Region-based multimodal image fusion using ICA bases. International Conference on Image Processing. Atlanta. 2006; 1801-1804.

[22] D Drajic, N Cvejic. Multimodal image fusion in presence of noise using sparse coding of ICA coefficients. IEEE International Symposium on Signal Processing and Information Technology, 2007: 343-346.

[23] V Petrovic, T Cootes. Information Representation for Image Fusion Evaluation. 9th international Conference on Information Fusion. 2006; 1-7. 